\title{
The Epidemiology of Clostridium difficile Infection in Children: A Population-Based Study
}

\author{
Sahil Khanna, ${ }^{1}$ Larry M. Baddour, ${ }^{2}$ W. Charles Huskins, ${ }^{3}$ Patricia P. Kammer, ${ }^{1}$ William A. Faubion, ${ }^{1}$ Alan R. Zinsmeister, ${ }^{4}$ \\ W. Scott Harmsen, ${ }^{4}$ and Darrell S. Pardi'
}

${ }^{1}$ Division of Gastroenterology and Hepatology, ${ }^{2}$ Division of Infectious Diseases, ${ }^{3}$ Division of Pediatric Infectious Diseases, and ${ }^{4}$ Division of Biomedical Statistics and Informatics, Mayo Clinic, Rochester, Minnesota

Background. The incidence of Clostridium difficile infection (CDI) is increasing, even in populations previously thought to be at low risk, including children. Most incidence studies have included only hospitalized patients and are thus potentially influenced by referral or hospitalization biases.

Methods. We performed a population-based study of CDI in pediatric residents (aged 0-18 years) of Olmsted County, Minnesota, from 1991 through 2009 to assess the incidence, severity, treatment response, and outcomes of CDI.

Results. We identified 92 patients with CDI, with a median age of 2.3 years (range, 1 month-17.6 years). The majority of cases $(75 \%)$ were community-acquired. The overall age- and sex-adjusted CDI incidence was 13.8 per 100000 persons, which increased 12.5-fold, from 2.6 (1991-1997) to 32.6 per 100000 (2004-2009), over the study period $(P<.0001)$. The incidence of community-acquired CDI was 10.3 per 100000 persons and increased $10.5-$ fold, from 2.2 (1991-1997) to 23.4 per 100000 (2004-2009) $(P<.0001)$. Severe, severe-complicated, and recurrent CDI occurred in $9 \%, 3 \%$, and $20 \%$ of patients, respectively. The initial treatment in $82 \%$ of patients was metronidazole, and $18 \%$ experienced treatment failure. In contrast, the initial treatment in $8 \%$ of patients was vancomycin and none of them failed therapy.

Conclusions. In this population-based cohort, CDI incidence in children increased significantly from 1991 through 2009. Given that the majority of cases were community-acquired, estimates of the incidence of CDI that include only hospitalized children may significantly underestimate the burden of disease in children.

Keywords. Clostridium difficile infection; epidemiology; pediatric; population-based; community-acquired.

Clostridium difficile infection (CDI) is a common cause of hospital- and community-acquired diarrhea. The incidence, severity, and likelihood of recurrence of CDI in adults have been defined in hospital- and population-based studies [1-5]. CDI has also been reported in populations previously considered to be at low risk, including young adults, pregnant females,

Received 16 October 2012; accepted 9 January 2013; electronically published 13 February 2013.

Correspondence: Sahil Khanna, MBBS, MS, Division of Gastroenterology and Hepatology, Mayo Clinic, 200 First St SW, Rochester, MN 55905 (khanna.sahil@ mayo.edu).

Clinical Infectious Diseases 2013;56(10):1401-6

(c) The Author 2013. Published by Oxford University Press on behalf of the Infectious Diseases Society of America. All rights reserved. For Permissions, please e-mail: journals.permissions@oup.com.

DOI: $10.1093 / \mathrm{cid} / \mathrm{cit} 075$ and children $[6,7]$. Traditionally, neonates and infants were believed to be asymptomatic carriers of $C$. difficile $[8,9]$; however, recent studies have suggested that $\mathrm{CDI}$ is emerging as a cause of diarrhea in infants and children [10-16]. This increasing incidence has been attributed, in part, to the emergence of a hypervirulent strain of $C$. difficile, increased antibiotic prescriptions, increased awareness of CDI among healthcare providers, and emergence of highly sensitive detection methodologies for CDI $[1,3]$. Most reports describing the incidence of CDI in children are based largely on hospitalized cohorts and are therefore subject to referral and hospitalization biases. There are no prior populationbased studies describing the epidemiology of CDI in children.

In this study, we assessed the incidence, severity, and treatment outcomes of CDI, including hospital- 
and community- acquired cases, in a population-based cohort of children.

\section{METHODS}

\section{Study Setting}

Using the resources of the Rochester Epidemiology Project (REP) $[17,18]$, we conducted a population-based cohort study of the epidemiology of CDI from 1991 through 2009 in residents aged $0-18$ years of Olmsted County, Minnesota. The REP provides access to all medical records, from all sources of care available to Olmsted County residents, and includes all medical diagnoses, hospital admissions, and surgical procedures, results of hematologic and laboratory tests, imaging tests, vaccinations, and drug prescriptions. A central diagnostic index links records from all outpatient visits, emergency room visits, hospitalizations, nursing home visits, surgical procedures, autopsy examinations, and death certificates for all residents since 1908. The REP therefore allows investigators to follow subjects through their outpatient and hospitalization contacts across all local medical facilities, regardless of where the care was delivered and of insurance status. In any 3-year period, $>95 \%$ of Olmsted County residents are examined at 1 of the 2 major healthcare systems in Olmsted County [17, 18]. The Mayo Clinic and Olmsted Medical Center institutional review boards approved this study.

\section{Case Definitions}

The REP database was searched for the ICD-9 (International Classification of Diseases, Ninth Revision) code for CDI (008.45), along with CDI as a microbiologic or clinical diagnosis (both inpatient and outpatient). After identification of possible cases of CDI, records of patients were reviewed. Clinical notes, laboratory results, and endoscopic and histopathologic reports were manually reviewed to confirm diagnoses. On the basis of recent recommendations [19], we defined cases of CDI as the occurrence of diarrhea ( $\geq 3$ loose stools per day) with a positive test for $C$. difficile toxin determined by enzyme immunoassay (EIA) or polymerase chain reaction (PCR), and no other identified causes of diarrhea. Patients who did not have diarrhea or who had other causes for diarrhea were excluded from the study $(n=7)$. The method for stool CDI testing changed from EIA to PCR in June 2007. All patient medical records were manually reviewed to confirm the diagnosis of CDI, identify separate cases of CDI in individual patients, and determine acquisition modality (community- vs hospital-acquired).

According to guidelines from the Infectious Diseases Society of America and the Society for Healthcare Epidemiology of America [19], CDI was defined as hospital-acquired if symptom onset occurred $>48$ hours after admission to, and $<4$ weeks after discharge from, a healthcare facility. CDI was defined as community-acquired if onset of symptoms occurred in the community or within 48 hours of admission to a hospital, provided symptom onset was $>12$ weeks after the last discharge from a hospital. CDI was defined as indeterminate if symptom onset occurred between 4 and 12 weeks after a hospital dismissal [20]. Patients with indeterminate infection $(\mathrm{n}=8)$ were considered as community-acquired in this study.

\section{Clinical Features, Treatment, and Outcomes}

We defined acid suppression medication use as the use of either a proton-pump inhibitor or a histamine-2 receptor blocker at the time of CDI diagnosis, and antibiotic exposure as the use of oral or parenteral antibiotics in the 12 weeks preceding CDI diagnosis. We defined inflammatory bowel disease as the presence of diagnosed ulcerative colitis, Crohn's disease, or indeterminate colitis, and malignancy as a current or previous diagnosis of a localized or metastatic solid tumor, lymphoma, or leukemia.

Severe CDI was defined as a peripheral white blood cell count $\geq 15000$ cells $/ \mathrm{mm}^{3}$ or a serum creatinine rise of $\geq 50 \%$ from baseline [19]. CDI was classified as severe-complicated if the infection was complicated with sepsis, hypotension, ileus, toxic megacolon, perforation, need for intensive care unit admission, surgery for a CDI-related complication (eg, megacolon, perforation, refractory colitis), or death $[19,20]$. Treatment failure was defined as the persistence of diarrhea or medication intolerance during antibiotic treatment leading to a change in treatment within 14 days. We defined recurrent CDI using the above-described case criteria if the onset of diarrhea occurred within 8 weeks of initial diagnosis and after documented symptom resolution $[19,20]$.

\section{Statistical Analyses}

The age- and sex-adjusted incidence of CDI per 100000 person-years was calculated (direct adjustment to the age and sex distribution of US whites, as of the 2000 census). We used Poisson regression models to evaluate the association of age, sex, and calendar period with crude incidence rates. Because the REP captures data on both inpatients and outpatients, calculations of both the overall and community-acquired incidence rates were done. The community-acquired rates also were adjusted to 2000 US census data as described above. Associations among demographics, clinical features, severity, and outcomes with mode of acquisition of CDI (ie, communityacquired vs hospital-acquired) were assessed.

\section{RESULTS}

\section{Incidence and Clinical Features}

Of the 99 patients identified, 7 were excluded because of lack of diarrhea or the presence of other identified causes of 
diarrhea. Of the remaining 92 CDI cases, $67 \%$ occurred in the last 3 years of the study. The median age at symptom onset was 2.3 years (range, 1 month to 17.6 years). The majority of cases $(54 \%)$ occurred in males. In the 12 weeks prior to CDI diagnosis, exposure to antibiotics occurred in 72 cases $(78 \%)$ and gastric acid suppression medications were used in 18 cases (20\%). Fifty-six (60.9\%) of all patients had antibiotic exposure, $2(2.2 \%)$ had exposure to gastric acid suppression medications alone, 16 (17.4\%) had exposure to both acid suppression medications and antibiotics, and 18 (19.5\%) had no exposure to either. Of all children with CDI, $4.3 \%(n=4)$ had inflammatory bowel disease and 5.5\% $(n=5)$ had an underlying malignancy.

Sixteen cases (17\%) occurred in children $<1$ year of age (median age, 8.5 months). Fifteen (93.8\%) were exposed to antibiotics and $5(31.3 \%)$ were exposed to gastric acid suppression medications.

The majority of cases (75\%) were community-acquired; only $25 \%$ of cases were hospital-acquired $(21.7 \%$ of patients had community onset of symptoms but had been hospitalized in the 4 weeks prior to the diagnosis of CDI, and $3.3 \%$ had onset of diarrhea after 48 hours of hospitalization). Only 15 patients $(16.3 \%)$ had neither preceding antibiotic exposure nor recent hospitalization prior to CDI. Of 69 patients with community-acquired CDI, $85.5 \%(n=59)$ had an outpatient or an emergency department visit in the 3 months prior to CDI onset, and 10 patients (10.9\% of all cases) did not have any healthcare exposure in the 12 weeks prior to CDI onset.

The overall age- and sex-adjusted incidence of CDI was 13.8 per 100000 person-years and increased from 2.6 per 100000
(1991-1997) to 5.7 per $100000(1998-2003)$ to 32.6 per 100000 (2004-2009) over the study period $(P<.001)$ (Figure $1 A$ ). The age-specific incidence was highest in the 0 - to 1-year age group, at 49.1 per 100000 person-years (Figure 1B).

The age- and sex-adjusted incidence of communityacquired CDI was 10.3 per 100000 person-years overall, and increased significantly over the study period, from 2.2 per 100000 (1991-1997) to 4.7 per 100000 (1998-2003) to 23.4 per 100000 (2004-2009) $(P<.001)$. The age-specific incidence was the highest in the 0 - to 1-year age group, at 43.6 per 100000 person-years. There was no association between sex and the overall incidence $(P=.57)$ or the incidence of communityacquired $\mathrm{CDI}(P=.75)$.

\section{Outcomes, Treatment Failure, and Recurrence}

Among the 69 patients with community-acquired CDI, only 9 (13\%) required hospitalization for management of CDI. Compared to children with hospital-acquired CDI, children with community-acquired CDI were younger, were less likely to have exposure to acid suppression medications, and were much less likely to develop severe infection (2.9\% vs $26.1 \%$; Table 1). There were no differences in outcomes among patients who were exposed to antibiotics alone, those who were exposed to antibiotics and gastric acid suppression medications, and those who had no exposure to acid suppression or antibiotics.

Initial treatment was with metronidazole in $81.5 \%$ of cases, oral vancomycin in $7.6 \%$, and rifaximin in $2.1 \%$. There were 13 treatment failures (18\%) with metronidazole and no treatment failures with vancomycin or rifaximin $(P=.11)$. There

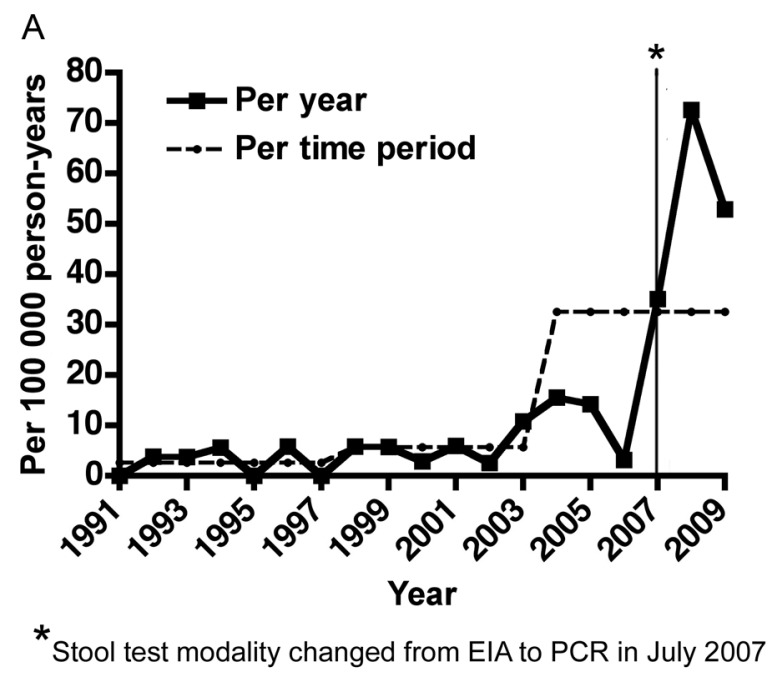

B

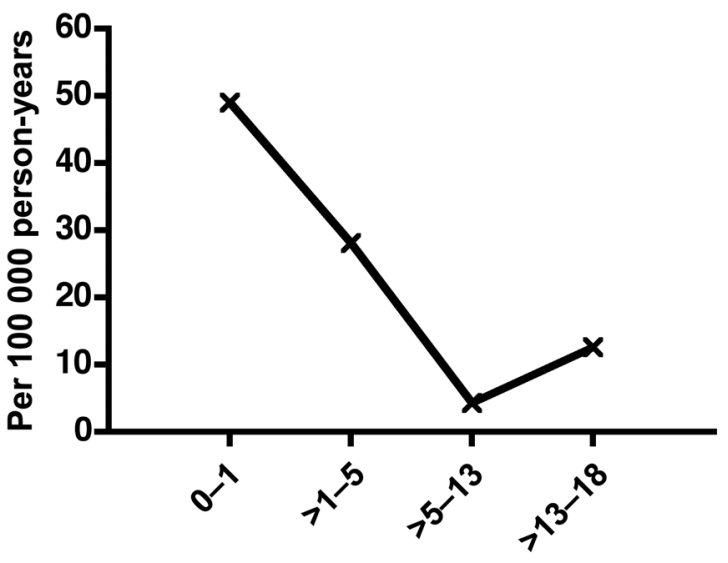

Age group (years)

Figure 1. Age- and sex-adjusted overall incidence of Clostridium difficile infection in children, 1991-2009. $A$, Trend in age- and sex-adjusted incidence rates over time for the overall study population. B, Age-specific incidence in the whole population. Abbreviations: ElA, enzyme immunoassay; PCR, polymerase chain reaction. 
Table 1. Comparison of Community-Acquired and HospitalAcquired Clostridium difficile Infection in Children, 1991-2009

\begin{tabular}{lccc}
\hline & $\begin{array}{c}\text { Community- } \\
\text { Acquired } \\
(\mathrm{n}=69)\end{array}$ & $\begin{array}{c}\text { Hospital- } \\
\text { Acquired } \\
(\mathrm{n}=23)\end{array}$ & PValue \\
Characteristic & $1.9(0.3-16.9)$ & $4.2(0.1-17.6)$ & .05 \\
\hline Median age, y (range) & $34(49.2)$ & $16(69.5)$ & .08 \\
Male sex & $52(75.4)$ & $20(86.9)$ & .22 \\
Antibiotic exposure & $10(14.5)$ & $8(34.9)$ & .04 \\
$\begin{array}{l}\text { Gastric acid suppression } \\
\quad \text { use }\end{array}$ & $2(2.9)$ & $6(26.1)$ & .001 \\
Severe CDI & $3(4.3)$ & $1(4.3)$ & .9 \\
Severe complicated CDI & $12(17.4)$ & $6(26.1)$ & .37 \\
Recurrent CDI & & & \\
\hline
\end{tabular}

Data are presented as No. (\%) unless otherwise specified.

Abbreviation: $\mathrm{CDI}$, Clostridium difficile infection.

was no treatment documented in $8.7 \%$, and these patients were managed conservatively. There was 1 patient with severe infection (as defined by laboratory criteria), and there were no complications or recurrences in patients who did not receive CDI-specific treatment.

In the entire cohort, there were 8 severe cases (8.7\%) and 4 severe-complicated cases (4.2\%), including 1 death in a patient with metastatic rhabdomyosarcoma. Of the 8 severe cases, 6 were initially treated with metronidazole and 1 with vancomycin, and 1 did not receive any documented treatment. The overall recurrence rate was $19.6 \%$ (21.3\% in children treated with metronidazole and $0 \%$ in children treated with oral vancomycin; $P=.07$ ). There were no differences in recurrence in those with severe CDI compared to mild to moderate CDI.

\section{DISCUSSION}

In this population-based epidemiologic study reporting the incidence of CDI in children, we found that CDI incidence increased significantly over the study period, consistent with findings described in other investigations [11, 21-23]. The uniqueness of our study is the inclusion of communityacquired cases that accounted for a majority of patients. Most of the prior studies of CDI in children included only hospitalacquired CDI cases. This limitation has important epidemiologic and clinical implications. Specifically, omission of these community-acquired cases would lead to underestimation of the overall incidence of CDI in children, and an overestimation of severity, given that hospital-acquired cases tend to be more severe than community-acquired cases. However, similar to the findings in a recent report from the Centers for Disease Control and Prevention, the majority of patients in our study with community-acquired CDI had healthcare exposure prior to CDI [24].
The largest prior study of CDI in children was hospital based, and it showed a significant increase in CDI incidence from 2001 to 2006 [10]. However, a distinction between hospital- and community-acquired CDI was not made. In that study, approximately one-fourth of patients were $<1$ year of age and the majority of patients (67\%) had underlying chronic medical conditions, which is higher than in our study [10].

An increasing incidence has also been described in adults and has been attributed to increasing antibiotic use and the presence of a hypervirulent strain [3,4]. However, we did not perform strain characterization in this investigation. Although these factors may play a role in the increase in incidence of CDI observed in our study, there also is the potential for diagnostic detection bias contributing to the increase in incidence in the latter time period of our study, as the stool assay for $C$. difficile at our institution changed from an EIA-based test to a more sensitive PCR-based test in July 2007. This more sensitive test could have led to increased detection and an apparent increase in incidence $[25,26]$. With increasing awareness of CDI among healthcare providers, it is also possible that increased testing for CDI could have led to an increased incidence.

The majority of our population (76\%) had prior antibiotic exposure, which has been noted in only $22 \%-57 \%$ of children with CDI in other studies $[11,15]$. A possible explanation for this difference is the availability of a centralized data linkage system that the REP provides, which captures all medications prescribed to all inhabitants of Olmsted County, regardless of clinical setting.

The highest incidence of CDI was seen in children aged $<1$ year. It is widely recognized that infants and young children can be colonized with $C$. difficile, and, therefore, some of the cases may represent colonization with diarrhea related to another cause [27]. However, CDI has been reported as a cause of diarrhea in infants in other studies [21,22], and in our study these patients did not have other identified causes of diarrhea despite thorough evaluation, and improved with treatment for CDI.

Severe infection was uncommon among communityacquired cases but was more common in the hospital-acquired group. Most patients were treated with metronidazole as the initial treatment followed by vancomycin, and there was no documented treatment failure in vancomycin. Recurrence rates were higher for metronidazole than vancomycin, although these were not statistically different. These findings are similar to a recent systematic review in adults, which demonstrated that treatment failure rates for metronidazole were $>1.5$ times higher than those for vancomycin, and there was no significant difference in recurrence rates [28]. Oral vancomycin has been demonstrated to be equivalent to metronidazole for mild to moderate CDI but superior to metronidazole for adults with severe CDI (cure rate, $97 \%$ for vancomycin vs $76 \%$ 
for metronidazole) [29]. In this study, the relapse rate was not significantly different between the 2 treatment groups [29]. However, larger, randomized, blinded studies stratified by severity are needed to evaluate possible differences in treatment outcomes between metronidazole and vancomycin in children.

The major strength of our study is that data have been collected over a period of 19 years from a stable population. Collecting data from a population-based cohort allowed us to study the epidemiology of CDI in both hospital- and communityacquired cohorts. However, we were unable to estimate the incidence of hospital-acquired CDI owing to lack of accurate information on hospital bed-days for children, especially since the cohort included newborns. Other limitations include the potential of missing data, such as laboratory tests used for defining severe CDI, and lack of information on C. difficile strain.

In conclusion, in this population-based cohort, CDI was uncommon in children from 1991 through 2009, although there was a significant increase in cases after 2006. Severe infection was infrequently seen and there were very few CDIrelated complications. A substantial fraction of cases occurred in patients managed as outpatients, which would have been missed if hospitalized children were the only identified cohort. Such an omission of community-acquired cases could result in an underestimation of disease incidence and overestimation of disease severity in children with CDI. In children presenting with diarrhea, CDI should be considered in the differential diagnosis, even in outpatients with an absence of recent hospitalization and antibiotic exposure.

\section{Notes}

Financial support. This work was supported by the Rochester Epidemiology Project (REP), which is supported by the National Institute on Aging of the National Institutes of Health (award number R01AG034676), and ViroPharma, which provided an unrestricted research grant for an investigator-initiated study to support data extraction, study coordinator time, and statistical analysis.

Disclaimer. The content is solely the responsibility of the authors and does not necessarily represent the official views of the National Institutes of Health. ViroPharma did not have direct access to the study data or statistical analysis. The study investigators performed all aspects of data collection, statistical analysis, and manuscript writing.

Potential conflicts of interest. All authors: No reported conflicts.

All authors have submitted the ICMJE Form for Disclosure of Potential Conflicts of Interest. Conflicts that the editors consider relevant to the content of the manuscript have been disclosed.

\section{References}

1. Kelly CP, LaMont JT. Clostridium difficile-more difficult than ever. N Engl J Med 2008; 359:1932-40.

2. Pepin J, Valiquette L, Alary ME, et al. Clostridium difficile-associated diarrhea in a region of Quebec from 1991 to 2003: a changing pattern of disease severity. CMAJ 2004; 171:466-72.

3. Khanna S, Pardi DS. The growing incidence and severity of Clostridium difficile infection in inpatient and outpatient settings. Expert Rev Gastroenterol Hepatol 2010; 4:409-16.
4. Khanna S, Pardi DS, Aronson SL, et al. The epidemiology of communityacquired Clostridium difficile infection: a population-based study. Am J Gastroenterol 2012; 107:89-95.

5. Khanna S, Pardi DS. Clostridium difficile infection: new insights into management. Mayo Clin Proc 2012; 87:1106-17.

6. Centers for Disease C, Prevention. Severe Clostridium difficile-associated disease in populations previously at low risk-four states, 2005. MMWR Morb Mortal Wkly Rep 2005; 54:1201-5.

7. Khanna S, Pardi DS, Aronson SL, Kammer PP, Baddour LM. Outcomes in community-acquired Clostridium difficile infection. Aliment Pharmacol Ther 2012; 35:613-8.

8. Al-Jumaili IJ, Shibley M, Lishman AH, Record CO. Incidence and origin of Clostridium difficile in neonates. J Clin Microbiol 1984; 19: 77-8.

9. Larson HE, Barclay FE, Honour P, Hill ID. Epidemiology of Clostridium difficile in infants. J Infect Dis 1982; 146:727-33.

10. Kim J, Smathers SA, Prasad P, Leckerman KH, Coffin S, Zaoutis T. Epidemiological features of Clostridium difficile-associated disease among inpatients at children's hospitals in the United States, 20012006. Pediatrics 2008; 122:1266-70.

11. Benson L, Song X, Campos J, Singh N. Changing epidemiology of Clostridium difficile-associated disease in children. Infect Control Hosp Epidemiol 2007; 28:1233-5.

12. Enoch DA, Butler MJ, Pai S, Aliyu SH, Karas JA. Clostridium difficile in children: colonisation and disease. J Infect 2011; 63:105-13.

13. Langley JM, LeBlanc JC, Hanakowski M, Goloubeva O. The role of Clostridium difficile and viruses as causes of nosocomial diarrhea in children. Infect Control Hosp Epidemiol 2002; 23:660-4.

14. Klein EJ, Boster DR, Stapp JR, et al. Diarrhea etiology in a children's hospital emergency department: a prospective cohort study. Clin Infect Dis 2006; 43:807-13.

15. Boenning DA, Fleisher GR, Campos JM, Hulkower CW, Quinlan RW. Clostridium difficile in a pediatric outpatient population. Pediatr Infect Dis 1982; 1:336-8.

16. Denno DM, Shaikh N, Stapp JR, et al. Diarrhea etiology in a pediatric emergency department: a case control study. Clin Infect Dis 2012; 55: 897-904.

17. Melton LJ 3rd. History of the Rochester Epidemiology Project. Mayo Clin Proc 1996; 71:266-74.

18. St Sauver JL, Grossardt BR, Yawn BP, Melton LJ 3rd, Rocca WA. Use of a medical records linkage system to enumerate a dynamic population over time: the Rochester Epidemiology Project. Am J Epidemiol 2011; 173:1059-68.

19. Cohen SH, Gerding DN, Johnson S, et al. Clinical practice guidelines for Clostridium difficile infection in adults: 2010 update by the Society For Healthcare Epidemiology of America (SHEA) and the Infectious Diseases Society of America (IDSA). Infect Control Hosp Epidemiol 2010; 31:431-55.

20. McDonald LC, Coignard B, Dubberke E, et al. Recommendations for surveillance of Clostridium difficile-associated disease. Infect Control Hosp Epidemiol 2007; 28:140-5.

21. Zilberberg MD, Shorr AF, Kollef MH. Increase in Clostridium difficilerelated hospitalizations among infants in the United States, 20002005. Pediatr Infect Dis J 2008; 27:1111-3.

22. Zilberberg MD, Tillotson GS, McDonald C. Clostridium difficile infections among hospitalized children, United States, 1997-2006. Emerg Infect Dis 2010; 16:604-9.

23. Rexach CE, Tang-Feldman YJ, Cantrell MC, Cohen SH. Epidemiologic surveillance of Clostridium difficile diarrhea in a freestanding pediatric hospital and a pediatric hospital at a university medical center. Diagn Microbiol Infect Dis 2006; 56:109-14.

24. Centers for Disease Control and Prevention. Vital signs: preventing Clostridium difficile infections. MMWR Morb Mortal Wkly Rep 2012; 61:157-62.

25. Sloan LM, Duresko BJ, Gustafson DR, Rosenblatt JE. Comparison of real-time PCR for detection of the $t c d C$ gene with four toxin 
immunoassays and culture in diagnosis of Clostridium difficile infection. J Clin Microbiol 2008; 46:1996-2001.

26. Khanna S, Pardi DS, Rosenblatt JE, Patel R, Kammer PP, Baddour LM. An evaluation of repeat stool testing for Clostridium difficile infection by polymerase chain reaction. J Clin Gastroenterol 2012; 46: $846-9$.

27. Jangi S, Lamont JT. Asymptomatic colonization by Clostridium difficile in infants: implications for disease in later life. J Pediatr Gastroenterol Nutr 2010; 51:2-7.
28. Vardakas KZ, Polyzos KA, Patouni K, Rafailidis PI, Samonis G, Falagas ME. Treatment failure and recurrence of Clostridium difficile infection following treatment with vancomycin or metronidazole: a systematic review of the evidence. Int J Antimicrob Agents 2012; 40: $1-8$.

29. Zar FA, Bakkanagari SR, Moorthi KM, Davis MB. A comparison of vancomycin and metronidazole for the treatment of Clostridium difficile-associated diarrhea, stratified by disease severity. Clin Infect Dis 2007; 45:302-7. 